\title{
Interpreting Relationships between the Concentrations of Plant Viruses and Numbers of Local Lesions
}

\author{
BY A. KLECZKOWSKI \\ Rothamsted Experimental Station, Harpenden, Hertfordshire
}

\begin{abstract}
SUMMARY: The effect of dilution on numbers of local lesions produced by plant viruses was tested graphically and statistically for compatibility with contrasting hypotheses. Experimental results are incompatible with the hypothesis that lesions are produced because of chance encounters between single virus particles and susceptible regions of a uniform type, and that variations in response to similar inoculation between different leaves or plants occur solely because of variations in numbers of such regions. The results are compatible with the hypothesis that individual susceptible regions vary in susceptibility so that different doses of virus are needed to produce a lesion (the variations being such that the logarithms of minimal effective doses are normally distributed). The second hypothesis is, therefore, more probably correct, but the first can be fitted to experimental results by introducing various auxiliary assumptions, such as the existence of qualitatively different virus particles and susceptible regions. Conclusive evidence is unlikely to come from mathematical treatment of existing results, but only from a new experimental approach.
\end{abstract}

There are two theories concerning the effect of concentration of virus inoculum on the production of a local lesion. According to one theory a local symptom develops as a result of the presence of at least one virus particle in the inoculum which has been suitably introduced into susceptible host tissue. This is analogous to the presence or absence of bacterial growth depending on the presence or absence of at least one viable bacterium in the volume of fluid inoculated upon a suitable medium. This theory does not allow for any variation in susceptibility between different susceptible regions of the host. Differences between hosts would arise wholly because of variation in numbers of susceptible regions.

The other theory assumes that susceptible regions vary in susceptibility so that the minimum virus concentration necessary to initiate local infection varies from one region to another.

Youden, Beale \& Guthrie (1935) and Bald (1937 $a, b, c)$ dealing mainly with tobacco mosaic virus, and Parker (1938) who worked with vaccinia virus, concluded that the numbers of lesions obtained with different dilutions of inocula approximately fit the equation derived from the Poisson series on the assumption that a local lesion is caused by the introduction into a 'susceptible region' of at least one virus particle. On the other hand, Bryan \& Beard (1940) concluded that their results with papilloma virus and Parker's results with vaccinia virus are better fitted by the assumption that regional susceptibility varies in such a way that the logarithm of minimal effective concentration is normally distributed. This is analogous to the susceptibility of animals to chemical reagents, such as drugs or poisons, which cause 'quantal' responses. Logarithms of minimal effective doses are usually normally distributed (Gaddum, 1933; Bliss, 1935; Finney, 1947). 
Lauffer \& Price (1945) considered both theories and concluded that the theory of local infection, caused by single virus particles entering uniformly susceptible regions, is compatible with experimental data obtained with all viruses so far investigated, whereas data obtained with plant viruses are definitely incompatible with the theory of variation in regional susceptibility. Their conclusions were not substantiated by statistical tests, but were based on approximate graphical fitting (judged by inspection) of experimental data to the curves of the equations derived from the two theories. As they point out, the $\chi^{2}$ test is inapplicable to results obtained with local lesions produced by plant viruses, although it can be, and has been, applied by Haldane (1939) to Parker's results with vaccinia virus, and by Bryan \& Beard (1940) to their results with papilloma virus. With these animal viruses the test was applicable because known numbers of susceptible sites were inoculated with known volumes of the inoculum, whereas with local lesions produced on plants inoculated by rubbing their leaves with virus solutions, neither is known. Dilution-infection series obtained with plant viruses, however, can be tested statistically for compatibility with theoretical assumptions by comparing two estimates of the same variance, one obtained from analysis of variance of all data in a given experiment, and the other from deviations of experimental from theoretical values. This can be done by making use of the fact that suitably transformed local-lesion counts are approximately normally distributed with a variance independent of the mean (Kleczkowski, 1949).

In the work described below, dilution-infection series were obtained with plant viruses and tested for their compatibility with each of two hypotheses. It was undertaken for three reasons. First, Lauffer \& Price's arguments for rejecting the hypothesis of host variation in regional susceptibility were considered inadequate. Secondly, it was considered that the published dilutioninfection series do not sufficiently cover the ranges of high virus concentration. Thirdly, it was thought desirable to analyse the results of at least a few experiments statistically. Published data could not be used for this purpose because the method employed necessitates knowing the individual lesion counts for each replication, whereas only the total numbers of lesions for each virus concentration have been published.

\section{EXPERIMENTAL}

Most of the experiments were made with tobacco mosaic virus (TMV) on Nicotiana glutinosa. Purified preparations of the virus were used in most experiments, but sap from infected tobacco plants was used in some. A few experiments were made with purified preparations of tomato bushy stunt virus (BSV) on $N$. glutinosa, and with the Rothamsted culture of tobacco necrosis virus (TNV) using sap from infected tobacco plants as inocula and bean plants (Phaseolus vulgaris, var. Prince), in the two primary leaves stage, as the test plant.

The plants were inoculated by rubbing the leaf surfaces with the forefinger wet with inoculum. Each dilution of the inoculum was rubbed on a number of half-leaves distributed in such a way as to eliminate as. many sources of 
variation as possible. The experimental design shown in Table 1 was used when eight different virus concentrations were tested on Nicotiana glutinosa. Each plant was trimmed to six comparable leaves. Each treatment had twelve replications and occurred three times in each of the four blocks of two plants,

Leaf

Table 1.
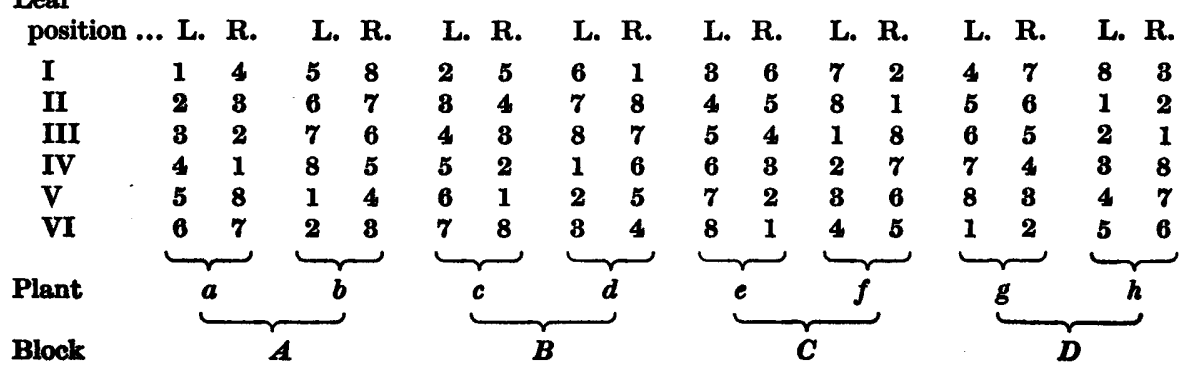

Table 2.

Leaf

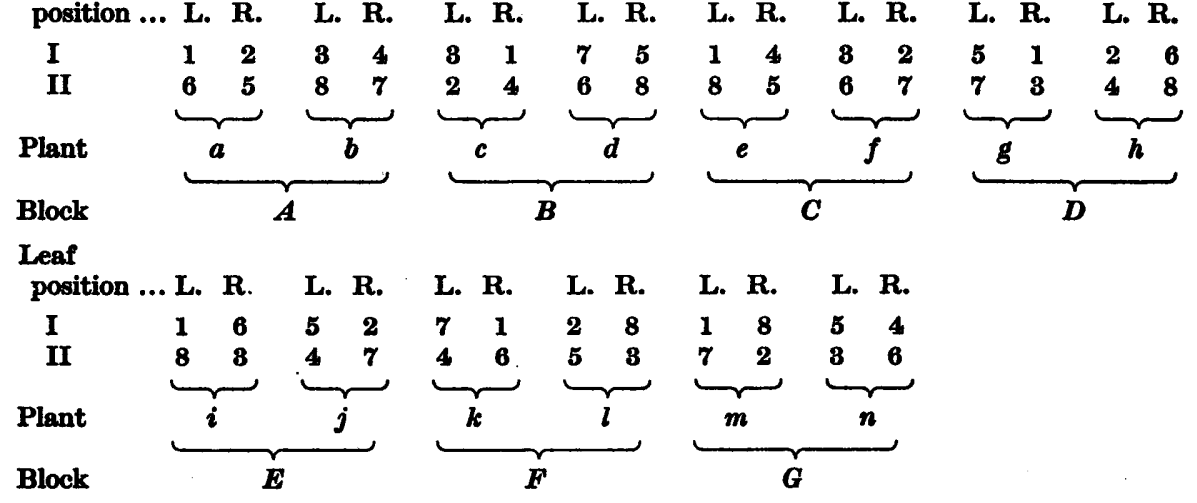

twice on each leaf position and six times each on the left (L.) and on the right (R.) half-leaf. When eight virus concentrations were tested on beans the experimental design shown in Table 2 was used. Only half of the whole design is shown. The other half is a repetition of the first but with the order L.-R. reversed. The two primary leaves of beans occupy the same position on the stem, giving one systematic variation fewer than with $N$. glutinosa. Each treatment had fourteen replications and occurred once in each of the fourteen blocks of two plants. Each treatment occurred with every other twice on one leaf, and four times on the same plant but on the opposite leaves.

\section{RESULTS}

\section{Graphical fittings}

In this section approximate graphical comparisons, judged by inspection, are made between experimental data and the curves of the equations based on the assumptions under test. In the next section the data of a few experiments are tested statistically for compatibility with the assumptions. 
The curves of the equations:

and

$$
\begin{aligned}
& Y=N\left(1-e^{-a x}\right), \\
& Y=N\left\{1-e^{-a x}(1+a x)\right\}, \\
& Y=N\left\{1-e^{-a x}\left(1+a x+\left[a^{2} x^{2} / 2 !\right]\right)\right\} \\
& Y=N\left\{1-e^{-a x}\left(1+a x+\left[a^{2} x^{2} / 2 !\right]+\left[a^{3} x^{3} / 3 !\right]\right)\right\}
\end{aligned}
$$

obtained from the Poisson series on the assumption that at least 1, 2, 3 and 4 virus particles, respectively, are necessary to produce a lesion in any of the $N$ accessible susceptible regions, are shown in Fig. $1 \mathrm{~A}$.

$\boldsymbol{Y}$ is the expected number of lesions per half-leaf, $N$ is the mean number of the 'susceptible regions' per half-leaf, $x$ is the virus concentration (in g./l.) or the dilution of infective sap, and $a$ is a constant. $N$ and $a$ are the parameters of the equations. The values of the parameters are unknown and have to be so adjusted for each equation to obtain the best possible fit to the experimental data.

Fig. 1B shows the curve of the equation

$$
Y=\lambda \frac{N}{\lambda \sqrt{(2 \pi)}} \int_{-\infty}^{t} \exp \left\{-\frac{1}{2}\left(\frac{t-\xi}{\lambda}\right)^{2}\right\} d t
$$

which corresponds to the assumption that 'susceptible regions' vary in susceptibility in such a way that logarithms of minimal virus concentrations, necessary to cause formation of a lesion, are normally distributed.

$Y$ is the expected number of lesions per half-leaf, $N$ is the mean number of 'susceptible regions' per half-leaf, $t=\log _{10} x(x=$ virus concentration in the inoculum or dilution of infective sap), $\xi=\log _{10} x_{0}\left(x_{0}=\right.$ virus concentration or dilution of infective sap when $50 \%$ of the susceptible regions develop lesions) and $\lambda$ is the standard deviation. This equation has three parameters, $N, \xi$ and $\lambda$, the values of which are unknown and have to be adjusted to give the best possible fit to the experimental data.

Table 3 gives the results of fifteen dilution-infection series, and their graphical fittings to equations ( $1 a$ ) and (2) are shown in Figs. 2-5.

In Exps. nos. 1-12 the inocula were either sap from infected plants or purified virus preparations with the highest concentration of $1 \mathrm{~g}$./1. or less. Figs. $2 \mathrm{~A}-4 \mathrm{~A}$ show that the results of some experiments, notably nos. 3, 5-9, 11 and 12, could be fitted satisfactorily to equation ( $1 a)$. (The deviation in Exp. no. 11, Fig. 4A, of the point corresponding to the highest virus concentration from the curve can be explained by the inhibitory effect of the undiluted sap used as inoculum.) The results of Exp. nos. 1, 2, 4 and 10 could not be fitted to equation (1a), as the curve slopes too steeply. The points of Exp. no. 10 (Fig. 4A) could be fitted satisfactorily by disregarding the point corresponding to the highest concentration.

In Exps. nos. 13-15 the highest virus concentration was $20 \mathrm{~g}$./1., and the results could not be fitted to equation ( $1 a$ ), the slope of the curve being too steep. The numbers of lesions were still increasing with increasing virus concentration up to a concentration of $20 \mathrm{~g}$. $/ \mathrm{l}$. 
Virus concentrations and numbers of lesions

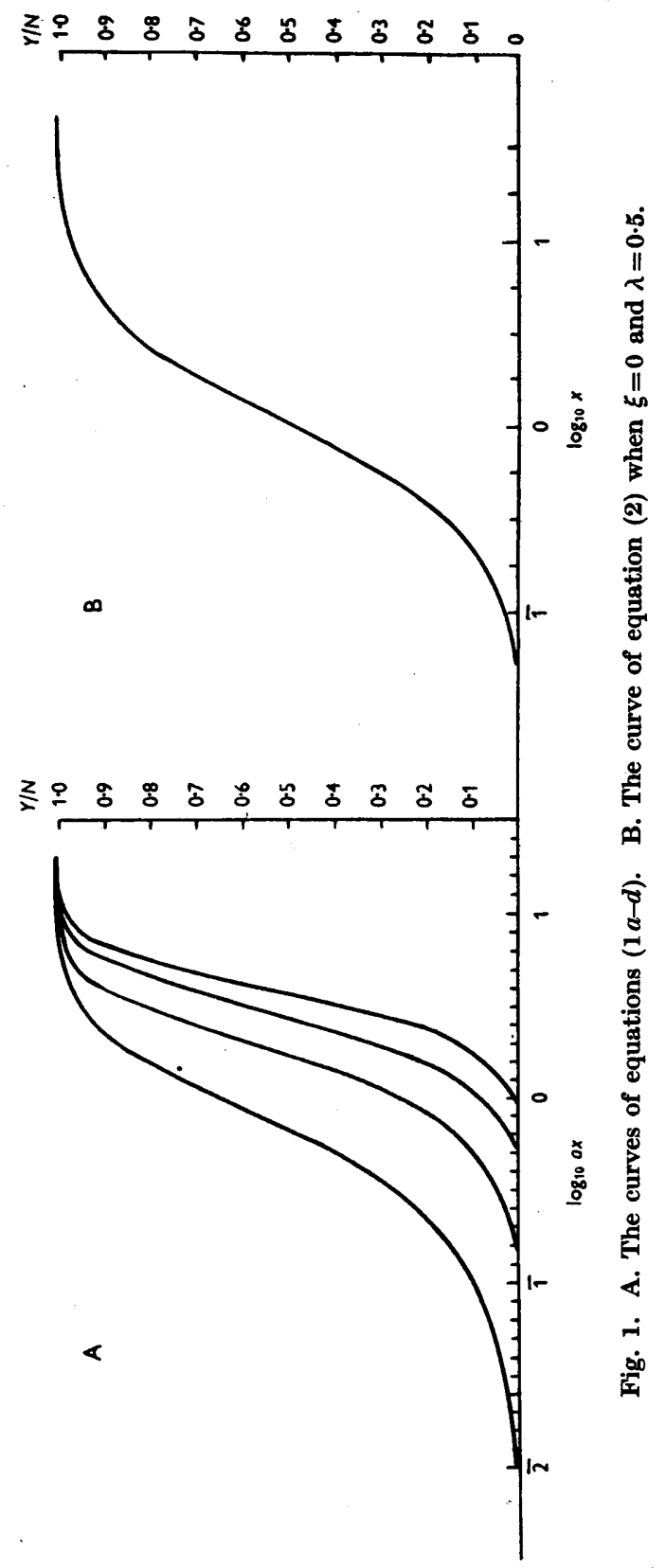


Several other dilution-infection series with the highest virus concentration at $20 \mathrm{~g}$./l. were made, and the results depended on the susceptibility of the test

Table 3. Numbers of lesions obtained by inoculating viruses at different concentrations

\begin{tabular}{|c|c|c|c|c|}
\hline $\begin{array}{c}\text { Exp. no. } 1 \mathrm{BSV} \\
\qquad \begin{array}{c}c=0 \mathrm{~g} \cdot / \mathrm{l} . \\
f=2 \\
n=24\end{array}\end{array}$ & $\begin{array}{c}\text { Exp. no. } 2 \text { TMV } \\
c=0 \cdot 2 \mathrm{~g} \cdot / \mathrm{l} . \\
f=2 \\
n=24\end{array}$ & $\begin{array}{c}\text { Exp. no. } 3 \text { TMV } \\
\begin{array}{c}c=0 \cdot 2 \text { g. } / \mathrm{l} . \\
f=2 \\
n=24\end{array}\end{array}$ & $\begin{array}{c}\text { Exp. no. } 4 \mathrm{TMV} \\
\begin{array}{c}c=\operatorname{sap} 1 / 2 \\
f=3 \cdot 16 \\
n=12\end{array}\end{array}$ & $\begin{array}{c}\text { Exp. no. } 5 \text { TMV } \\
\begin{array}{c}c=1 \cdot 0 \mathrm{~g} \cdot / \mathrm{l} \\
f=3 \cdot 16 \\
n=12\end{array}\end{array}$ \\
\hline 3491 & 8482 & 769 & 1525 & 2298 \\
\hline $\mathbf{8 3 4 4}$ & 2970 & 742 & 1288 & 2285 \\
\hline 2829 & 2641 & 423 & 722 & 1650 \\
\hline 2588 & 1674 & 282 & 551 & 989 \\
\hline 1256 & 1877 & 188 & 362 & 511 \\
\hline 1055 & 791 & 121 & 207 & 367 \\
\hline 898 & 600 & 69 & 67 & 67 \\
\hline 618 & 378 & 34 & 42 & 42 \\
\hline 414 & 193 & 12 & & \\
\hline 281 & 81 & 18 & & \\
\hline 121 & 97 & 10 & & \\
\hline 41 & 42 & 3 & & \\
\hline
\end{tabular}

Exp. no. 6 TMV Exp. no. 7 TMV Exp. no. 8* TMV Exp. no. 9 TMV Exp. no. 10 TMV

\begin{tabular}{|c|c|c|c|c|}
\hline $\begin{array}{l}c=\operatorname{sap} 1 / 5 \\
f=3 \cdot 16 \\
n=12\end{array}$ & $\begin{array}{l}c=\operatorname{sap~} 1 / 5 \\
f=3 \cdot 16 \\
n=12\end{array}$ & $\begin{array}{l}c=0 \cdot 3 \mathrm{~g} \cdot / \mathrm{l} . \\
f=4 \\
n=12\end{array}$ & $\begin{aligned} c & =1 \cdot 0 \mathrm{~g} . / 1 . \\
f & =3 \cdot 16 \\
n & =12\end{aligned}$ & $\begin{array}{l}c=1 \cdot 0 \mathrm{~g} . / 1 \\
f=3 \cdot 16 \\
n=12\end{array}$ \\
\hline 1216 & 1066 & $\mathbf{3 4 5 3}$ & 1097 & 3848 \\
\hline 1052 & 489 & 1589 & 910 & 2747 \\
\hline 621 & 219 & 914 & 677 & 2603 \\
\hline 253 & 166 & 270 & 396 & 2063 \\
\hline 189 & 99 & 94 & 160 & 868 \\
\hline 72 & 61 & 23 & 70 & 529 \\
\hline 18 & 12 & 5 & $\mathbf{3 8}$ & 160 \\
\hline 8 & $\theta$ & 4 & 7 & 58 \\
\hline
\end{tabular}

Exp. no. 11 TNV Exp. no. 12 TNV Exp. no. 13 TMV Exp. no. 14 TMV Exp. no. 15 TMV

\begin{tabular}{|c|c|c|c|c|}
\hline $\begin{array}{l}c=\text { undil. sap } \\
f=8 \cdot 16 \\
n=14\end{array}$ & $\begin{array}{l}c=\text { undil. sap } \\
f=3 \cdot 16 \\
n=14\end{array}$ & $\begin{array}{l}c=20 \cdot 0 \mathrm{~g} \cdot / \mathrm{l} \\
f=5 \\
n=12\end{array}$ & $\begin{array}{l}c=20 \cdot 0 \mathrm{~g} \cdot 1 \mathrm{l} \\
f=5 \\
n=12\end{array}$ & $\begin{array}{l}c=20 \cdot 0 \mathrm{~g} \cdot / \mathrm{l} . \\
f=3 \cdot 16 \\
n=12\end{array}$ \\
\hline 1790 & 708 & 2906 & 1426 & 826 \\
\hline 2055 & 632 & 2510 & 1162 & 808 \\
\hline 1403 & 414 & 1626 & 653 & 590 \\
\hline 921 & 208 & 1484 & $\mathbf{4 6 1}$ & 478 \\
\hline $\mathbf{3 4 3}$ & 91 & 972 & 179 & 376 \\
\hline 133 & 41 & 628 & 82 & 174 \\
\hline 88 & 5 & 276 & 43 & 152 \\
\hline \multirow[t]{2}{*}{15} & 4 & 146 & 8 & 122 \\
\hline & & & & 30 \\
\hline
\end{tabular}

$c=$ inoculum containing the highest virus concentration in a given experiment.

$f=$ factor by which other inocula were consecutively diluted.

$n=$ number of half-leaves on which given numbers of lesions were obtained.

* The leaves were sprinkled with 'Celite' (diatomaceous silica Filter-Aid, Johns-Manville) before inoculation in order to increase the numbers of lesions formed.

plants used. With less susceptible plants the results resembled those shown in Fig. 5. The leaves of more susceptible plants were covered with coalescing 


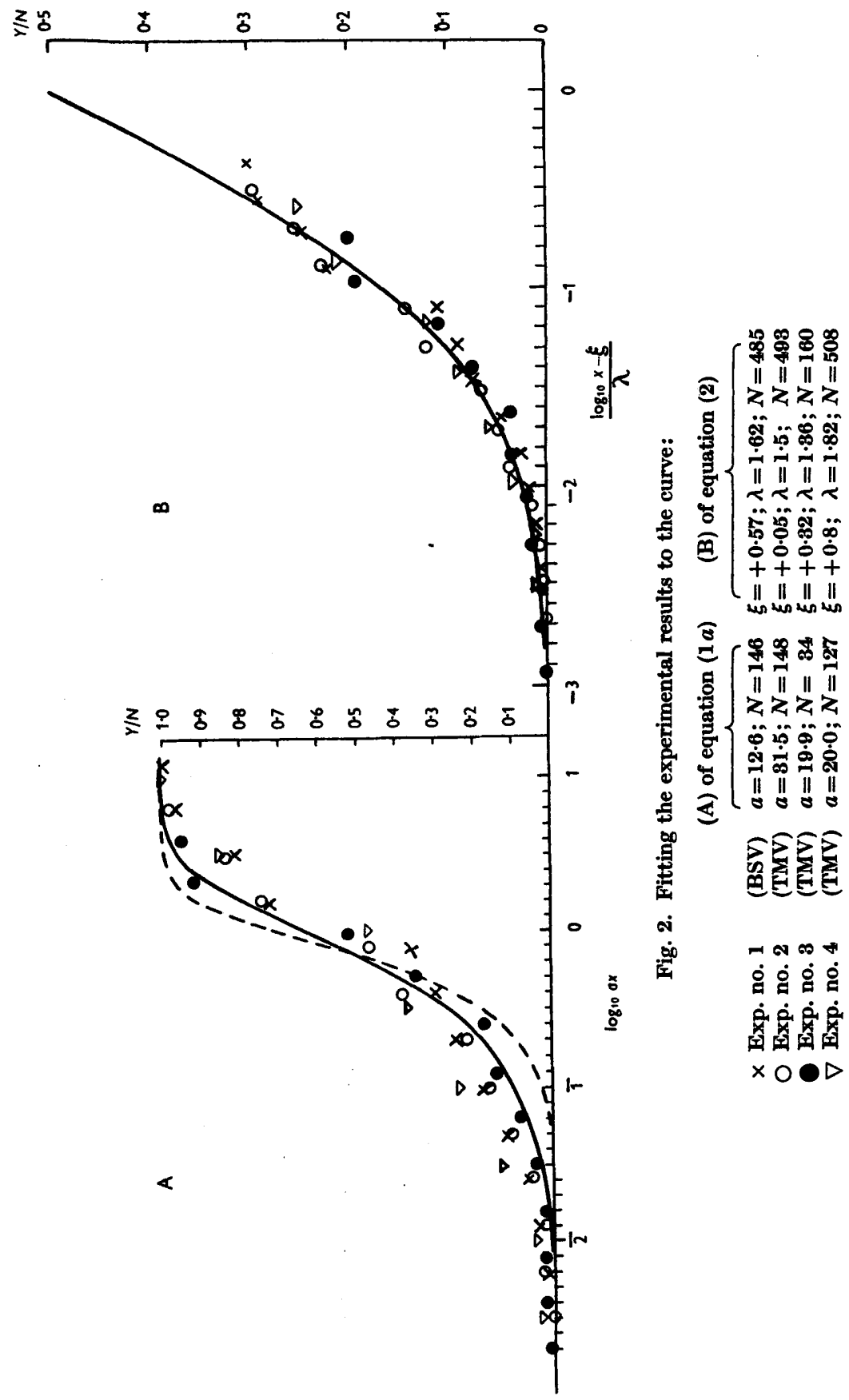




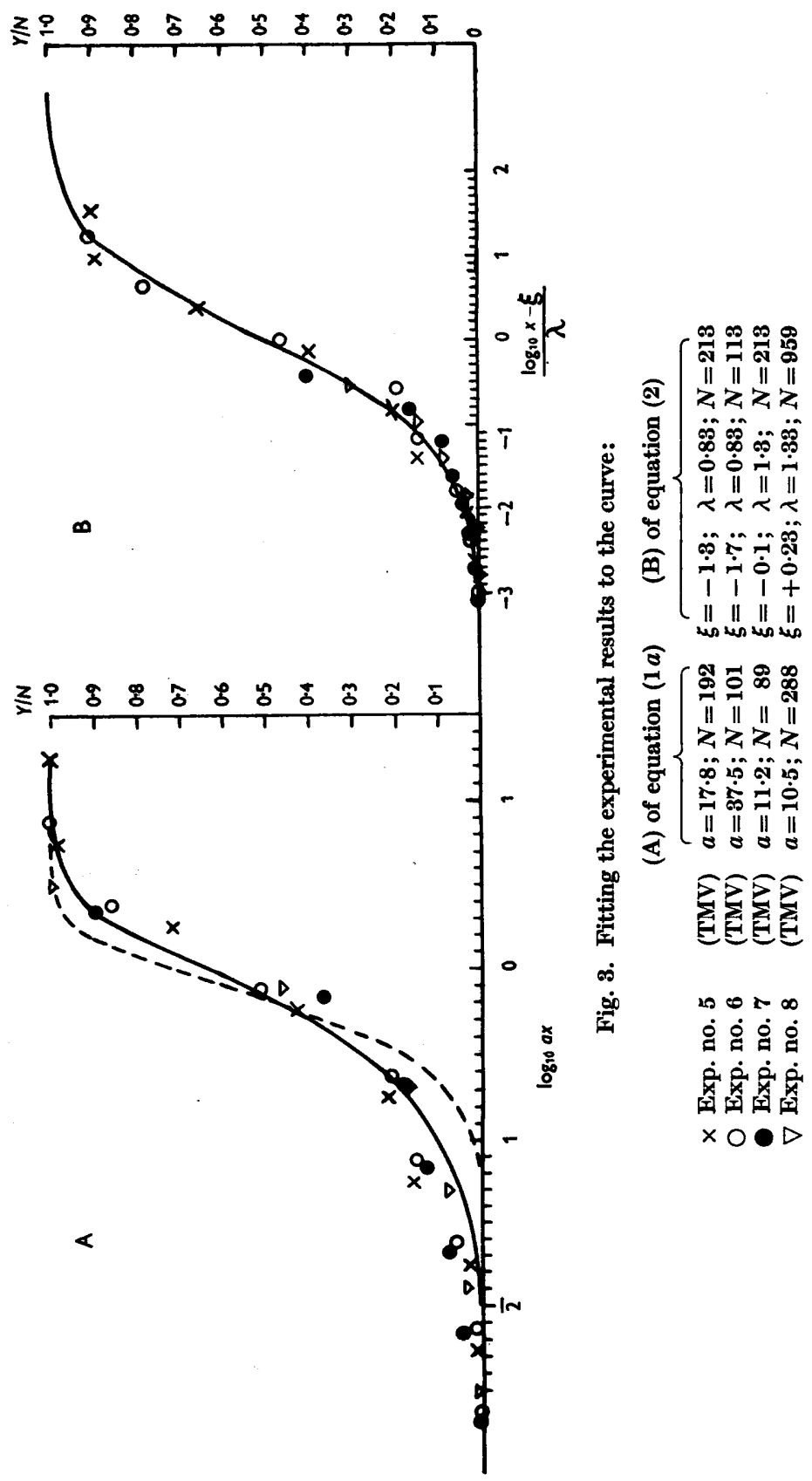




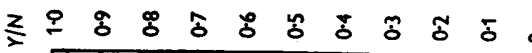

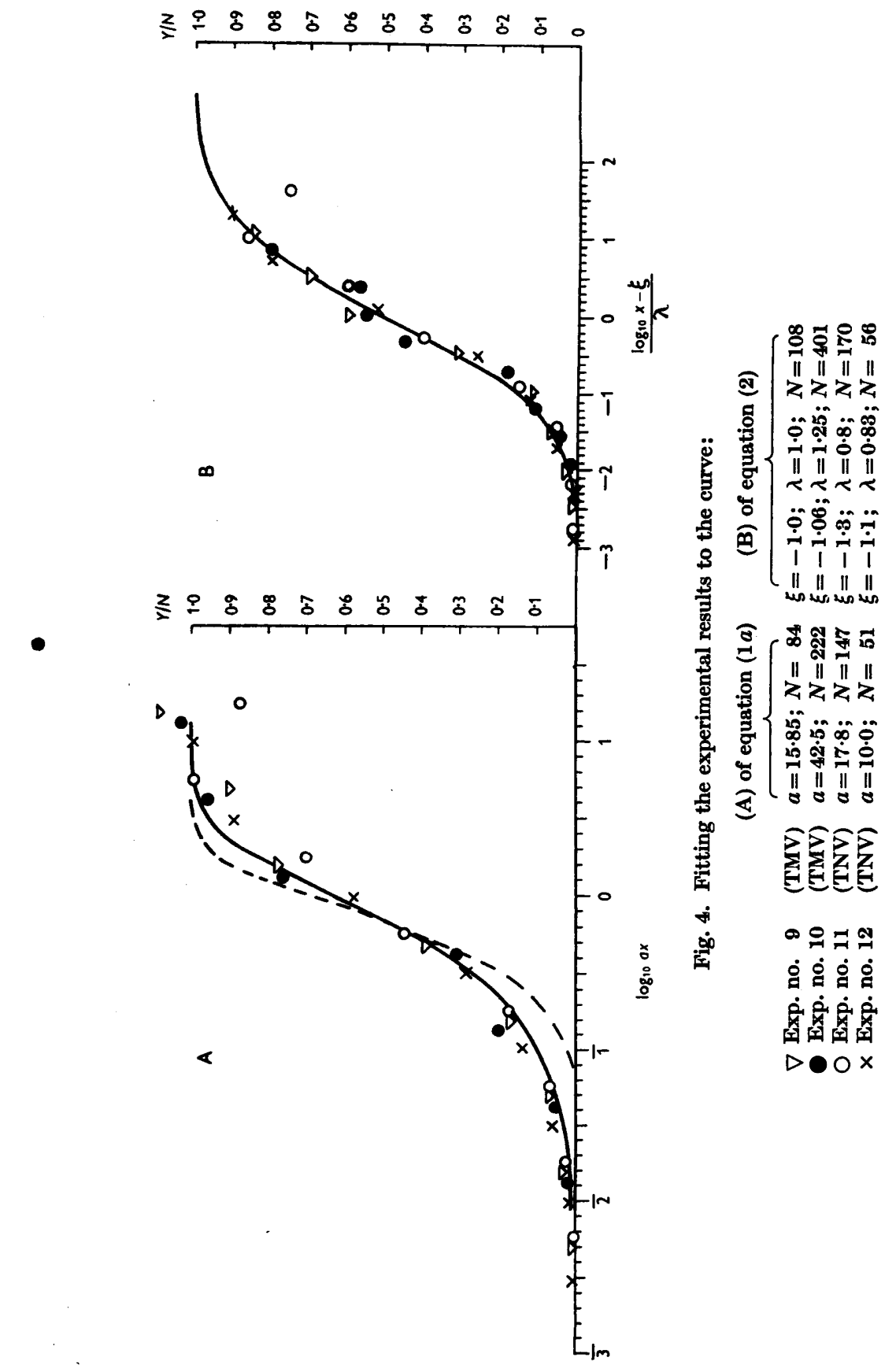


lesions when the virus concentration was much less than 20 g./l. As these lesions were uncountable, no comparisons with any of the curves could be made.

- The curve shown by broken lines in Figs. 2A-4A is that of equation (1 b) based on the assumption that at least two virus particles are needed to cause a lesion. The curve is placed so that its $50 \%$ point coincides with that of the curve of equation ( $1 a$ ). The slope of this curve is obviously too steep to fit any of the experimental data shown in Figs. 2-5. This also excludes the possibility

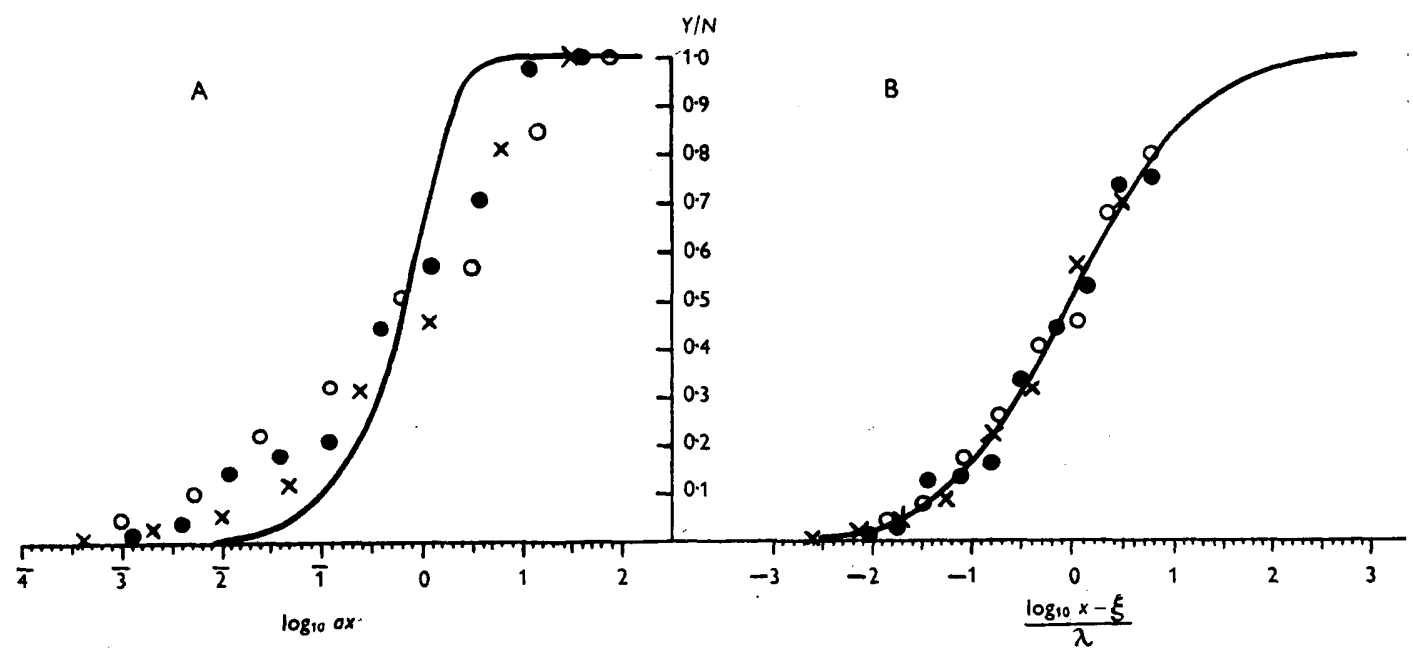

Fig. 5. Fitting the experimental results to the curve:

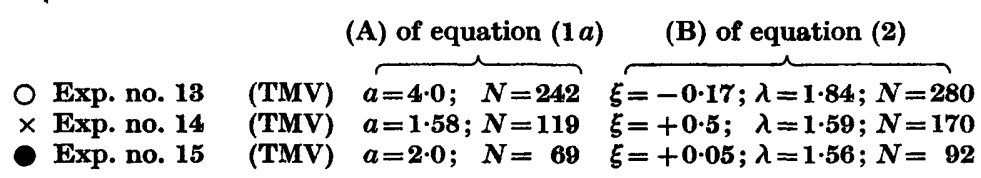

of fitting the curves of the equations for at least three, four or more particles, as their slopes are progressively steeper.

Figs. 2B-5B show that the results of all the experiments described here could be fitted approximately to equation (2), based on the assumption that the logarithm of the minimal effective virus concentration is normally distributed. The deviation of the point corresponding to the highest concentration of TNV in Exp. no. 11, Fig. $4 \mathrm{~B}$, from the curve can be explained, as previously remarked, by the inhibiting effect of undiluted sap used as inoculum.

\section{Statistical analysis}

A value $c$ can be found such that the value $z=\log _{10}(y+c)$, where $y$ is the number of lesions per half-leaf, is approximately normally distributed with a standard error independent of the mean (Kleczkowski, 1949). Statistical analyses of some of the dilution-infection series were made using this transformation. A preliminary value of $c$ to be used for the results of a particular 
experiment was estimated by using the regression equation of standard errors of the numbers of lesions per half-leaf for each virus dilution on the mean number for the same dilution. The value of $c$ is equal to the distance (in the negative direction) from the origin to the point of intersection of the regression line with the axis of abscissae. This procedure was repeated using the transformed value $z=\log _{10}(y+c)$ instead of $y$ for the estimation of standard errors for each virus dilution. If a regression line, almost parallel to the axis of the abscissae was obtained, the value of $c$ was considered satisfactory; otherwise it was suitably readjusted.

When a hypothesis leading to any of the equations $(1 a-d)$ or $(2)$ is tested, the value $Y_{i}=\phi\left(x_{i}\right)$ given by the equation is assumed to be the theoretical arithmetic mean of numbers of lesions per half-leaf corresponding to a given virus concentration $x_{i}$, whereas the mean values of $z=\log _{10}(y+c)$ are logarithms of geometric means of the experimental values $(y+c)$. The theoretical arithmetic mean has, therefore, to be transformed into the logarithm of geometric mean, which can then be compared with the mean value of $z$.

If the logarithms of the population of values $(y+c)$ are normally distributed with the mean $\mu$ and the variance $\sigma^{2}$, the arithmetic mean $Y$ plus the constant $c$ is

$$
\begin{aligned}
Y+c & =E\left(10^{z}\right)=\frac{1}{\sigma \sqrt{ }(2 \pi)} \int_{-\infty}^{\infty} 10^{z} \exp \left\{-\frac{1}{2 \sigma^{2}}(z-\mu)^{2}\right\} d z \\
& =10^{\mu} e^{\mathbf{z}(2 \cdot 3 \sigma)^{2}}=M_{\sigma} e^{2.645 \sigma^{2}},
\end{aligned}
$$

where $M_{g}=10^{\mu}$ is the theoretical geometric mean. Thus

$$
\log _{10} M_{\sigma}=\log _{10}(Y+c)-1 \cdot 149 \sigma^{2} \text {. }
$$

The value of $\sigma^{2}$ is unknown, but its estimate, $s^{2}$, is obtained from the analysis of variance of the experimental results transformed into $z=\log (y+c)$. Thus the theoretical value of $\log _{10} M_{g}$ will be approximately given by

so that $E(Z)=\log _{10} M_{g}$.

$$
Z=\log _{10}(Y+c)-1 \cdot 149 s^{2}
$$

The parameters of the equations $Y=\phi(x)$ were adjusted by the method of least squares, i.e. by minimizing the value $\Sigma\left(\bar{z}_{i}-Z_{i}\right)^{2}$, where $\bar{z}_{i}$ 's are the sample means of $n$ values of $z_{i}$ for each virus concentration $x_{i}$ and $Z_{i}$ 's are the values of $Z$ for the same virus concentration, obtained from equation (3). As all $\bar{z}_{i}$ 's are assumed to have the same variance, this procedure is equivalent to the method of maximum likelihood.

If the assumptions on which the function $Y=\phi(x)$ is based are true, the value $\frac{n}{W} \Sigma\left(\bar{z}_{i}-Z_{i}\right)^{2}$ will give an estimate of the variance $\sigma^{2}$, based on $W$ degrees of freedom, independently of that given by $s^{2}$, obtained from the analysis of variance. The number of the degrees of freedom, $W$, is equal to the number of treatments (i.e. different virus concentrations) minus the number of adjustable parameters in the equation $Y=\phi(x)$. Whether or not the two estimates of $\sigma^{2}$ are compatible is tested by the z-test or by the variance ratio test. When the ratio of the greater to the smaller estimate was found significant, the assumptions on which the tested function $Y=\phi(x)$ is based were considered incompatible with the experimental data. 
Only treatments that gave a mean number of not less than five lesions per half-leaf could be analysed in this way, for if the means are smaller the transformation $z=\log _{10}(y+c)$ cannot be expected to make the variance independent of the mean (Kleczkowski, 1949).

The results of only three experiments (nos. 9, 10 and 13) were analysed because the analysis is too time-consuming. The three were chosen because each gave results of different type. All fitted approximately to the curve of equation (2). Only one (no. 9) fitted to the curve of the equation (1a). No. 13 obviously did not fit to it, and no. 10 could be fitted only if the point corresponding to the highest virus concentration was disregarded.

Table 4. Analyses of variances

\begin{tabular}{|c|c|c|c|c|c|c|c|c|c|}
\hline \multirow[t]{2}{*}{ Transformation $\rightarrow$} & \multicolumn{3}{|c|}{$\begin{array}{c}\text { Exp. no. } 9 \\
z=\log _{10}(y+3)\end{array}$} & \multicolumn{3}{|c|}{$\begin{array}{c}\begin{array}{c}\text { Exp. no. } 10 \\
z=\log _{10}(y+20)\end{array} \\
.\end{array}$} & \multicolumn{3}{|c|}{$\begin{array}{c}\text { Exp. no. } 13 \\
z=\log _{10}(y+20)\end{array}$} \\
\hline & D.F. & $\begin{array}{c}\text { Sums } \\
\text { of } \\
\text { squares }\end{array}$ & $\begin{array}{c}\text { Mean } \\
\text { squares } \\
\left(s^{2}\right)\end{array}$ & D.F. & $\begin{array}{c}\text { Sums } \\
\text { of } \\
\text { squares }\end{array}$ & $\begin{array}{c}\text { Mean } \\
\text { square } \\
\left(s^{2}\right)\end{array}$ & D.F. & $\begin{array}{c}\text { Sums } \\
\text { of } \\
\text { squares }\end{array}$ & $\begin{array}{c}\text { Mean } \\
\text { square } \\
\left(s^{2}\right)\end{array}$ \\
\hline Between treatments & 5 & 9.9611 & & 7 & $14 \cdot 1082$ & & 7 & $\mathbf{8 \cdot 9 7 2 7}$ & \\
\hline Between blocks & $\mathbf{3}$ & 0.6217 & & $\mathbf{3}$ & 0.4708 & & $\mathbf{3}$ & $2 \cdot 0667$ & \\
\hline Between leaf positions & - & - * & & 5 & 0.9951 & & $\mathbf{5}$ & 1.0180 & \\
\hline Residual & 63 & $2 \cdot 9854$ & 0.0474 & 80 & $2 \cdot 9825$ & 0.0873 & 80 & $2 \cdot 2305$ & $0 \cdot 0286$ \\
\hline Total & 71 & $18 \cdot 5682$ & & 95 & $\mathbf{1 8} \cdot \mathbf{5 5 6 6}$ & & 95 & $14 \cdot 2879$ & \\
\hline
\end{tabular}

* The sum of squares was 0.1004 with 5 degrees of freedom. No precision would be gained by including this in the analysis of variance.

The analyses of variances for the three experiments are given in Table 4. All the experiments had eight treatments (virus concentrations) with twelve replications of each, and the experimental design was that shown above. In experiment no. 9 the two lowest virus concentrations gave mean numbers of less than five lesions per half-leaf so the results obtained with these were not used. All the data obtained in the two other experiments were used.

Tables 5-7 give the results of comparisons of the experimental values of $\bar{z}$ of Exp. nos. 9, 10 and 13 with computed values of $Z$, obtained from equation (3) by substitution values for $Y$ obtained from the equations $(1 a, b)$ and (2), the parameters of which were adjusted by the method of least squares. The equation

$$
Y=N_{1}\left(1-e^{-a_{1} x}\right)+N_{2}\left(1-e^{-a_{2} x}\right)
$$

was also tested with the results of Exp. no. 13, the meaning of which is discussed later.

Equation ( $1 a$ ) could be fitted satisfactorily to the results of Exp. 9 but not to those of Exp. 10. It could be fitted to no. 10 only if the result obtained with the highest virus concentration was disregarded. Equation (1b) does not fit the results of Exps. nos. 9 and 10, even if the result obtained in Exp. no. 10 with the highest virus concentration was disregarded. Fig. 5A shows that equations ( $1 a$ and $b$ ) obviously do not fit the results of Exp. no. 13, so no statistical test of fit of these equations was made. Equation (2) can be fitted satisfactorily to the results of all three experiments. It is also shown that equation (4) can be fitted to the results of Exp. no. 13. 


\section{DISCUSSION}

The fact that equation ( $1 a$ ) did not fit the results of all the experiments must alone be considered sufficient for the conclusion that the assumptions from which it was derived is false. There are, however, other reasons for its rejection.

Table 5. Comparisons of experimental with computed values for Exp. no. 9

\begin{tabular}{|c|c|c|c|c|c|c|c|}
\hline \multirow{2}{*}{$\begin{array}{l}\text { TMV } \\
\text { conc. } \\
\text { (g./1.) }\end{array}$} & \multirow{2}{*}{$Y=\underset{\bar{z}}{\phi}(x) \rightarrow$} & \multirow{2}{*}{$\begin{array}{c}I \\
(1 a) \\
Z\end{array}$} & \multirow{2}{*}{$\begin{array}{c}\text { II } \\
(\mathbf{1} b) \\
Z\end{array}$} & \multirow{2}{*}{$\begin{array}{c}\text { III } \\
\text { (2) } \\
Z\end{array}$} & \multicolumn{3}{|c|}{ Parameters } \\
\hline & & & & & I & II & III \\
\hline $\begin{array}{l}1.0 \\
0.816 \\
0 \cdot 1 \\
0.0816 \\
0.01 \\
0.00816\end{array}$ & $\begin{array}{l}1.9433 \\
1.8250 \\
1 \cdot 7000 \\
1.4883 \\
1 \cdot 1525 \\
0 \cdot 8942\end{array}$ & $\begin{array}{l}1.8366 \\
1 \cdot 8358 \\
1 \cdot 7761 \\
1 \cdot 5260 \\
1 \cdot 1649 \\
0 \cdot 8261\end{array}$ & $\begin{array}{l}1 \cdot 7821 \\
1 \cdot 7821 \\
1 \cdot 7816 \\
1 \cdot 6793 \\
\mathbf{1 . 2 0 8 0} \\
\mathbf{0 . 6 6 8 7}\end{array}$ & $\begin{array}{l}1.9187 \\
1.8388 \\
1.6961 \\
1.4791 \\
1.1892 \\
0.8639\end{array}$ & $\begin{array}{l}a=20 \\
N=74.7\end{array}$ & $\begin{array}{r}a=00 \cdot 6 \\
N=65.5\end{array}$ & $\begin{aligned} \xi & =-1 \cdot 025 \\
\lambda & =0 \cdot 0 \\
N & =104\end{aligned}$ \\
\hline$\frac{n}{W} \Sigma\left(\vec{z}_{i}-Z_{i}\right)^{2} \rightarrow$ & $W \rightarrow$ & $\begin{array}{c}4 \\
0.0705\end{array}$ & $\begin{array}{c}4 \\
0.3790\end{array}$ & $\begin{array}{c}\mathbf{3} \\
0.0128\end{array}$ & & & \\
\hline \multicolumn{2}{|c|}{$\begin{array}{l}\text { Variance ratio } \\
\text { D.F. } n_{1} \\
n_{2}\end{array}$} & $\begin{array}{r}1 \cdot 487 \\
4 \\
63\end{array}$ & $\begin{array}{c}8.015^{*} \\
4 \\
63\end{array}$ & $\begin{array}{r}\mathbf{3} \cdot 854 \\
63 \\
8\end{array}$ & & & \\
\hline
\end{tabular}

Table 6. Comparisons of experimental with computed values for Exp. no. 10

\begin{tabular}{|c|c|c|c|c|c|c|c|c|c|}
\hline \multirow{2}{*}{$\begin{array}{l}\text { TMV } \\
\text { conc. } \\
\text { (g./l.) }\end{array}$} & \multirow{2}{*}{\multicolumn{2}{|c|}{$Y=\underset{\bar{z}}{\phi}(x) \rightarrow \underset{Z}{(1 / a)}$}} & \multirow{2}{*}{$\underset{\mathbf{( 1 a )}}{\mathrm{II}}$} & \multirow{2}{*}{$\underset{Z}{\text { III }}$} & \multirow{2}{*}{$\begin{array}{l}\text { IV } \\
\text { (2) } \\
Z\end{array}$} & \multicolumn{4}{|c|}{ Parameters } \\
\hline & & & & & & I & II & III & IV \\
\hline $\begin{array}{l}.0 \\
.816 \\
.1\end{array}$ & $\begin{array}{l}2 \cdot 4967 \\
2 \cdot 8275 \\
2 \cdot 2883\end{array}$ & $\begin{array}{l}\mathbf{2 \cdot 3 6 9 1} \\
\mathbf{2 \cdot 8 6 9 1} \\
\mathbf{2 \cdot 8 5 8 3}\end{array}$ & $\begin{array}{c}-\overline{2 \cdot 2969} \\
2 \cdot 2958\end{array}$ & $\begin{array}{c}-\overline{2398} \\
2 \cdot 2398\end{array}$ & $\begin{array}{l}\mathbf{2} \cdot 4482 \\
\mathbf{2} \cdot \mathbf{3 9 3 9} \\
\mathbf{2 \cdot 2 9 2 0}\end{array}$ & $\begin{aligned} a & =47 \\
N & =284\end{aligned}$ & $\begin{aligned} a & =59 \\
N & =108.5\end{aligned}$ & $\begin{aligned} a & =253 \\
N & =172\end{aligned}$ & $\begin{aligned} \xi & =-1 \cdot 82 \\
\lambda & =1 \cdot 01 \\
N & =320\end{aligned}$ \\
\hline .0816 & $2 \cdot 2183$ & $2 \cdot 2608$ & 2.2809 & $2 \cdot 2386$ & $2 \cdot 1541$ & & & & \\
\hline & 1.9062 & 1.9895 & 1.0926 & $2 \cdot 1153$ & 1.9577 & & & & \\
\hline .00316 & $1 \cdot 7592$ & 1.6754 & 1.6877 & 1.6796 & 1.7268 & & & & \\
\hline .001 & 1.4958 & 1.4445 & $1 \cdot 4533$ & 1.8514 & 1.5083 & & & & \\
\hline .000316 & $1 \cdot \mathbf{3 8 8 3}$ & 1.3265 & $1 \cdot 8510$ & $1 \cdot 2688$ & $\mathbf{1} \cdot \mathbf{3 6 4 1}$ & & & & \\
\hline \multicolumn{2}{|c|}{$W \rightarrow$} & 6 & 5 & 5 & $\mathbf{5}$ & & & & \\
\hline \multicolumn{2}{|c|}{$\frac{i}{\bar{V}} \Sigma\left(\bar{z}_{i}-Z_{i}\right)^{2} \rightarrow$} & 0.0936 & 0.0408 & 0.2296 & 0.0852 & & & & \\
\hline \multicolumn{2}{|c|}{ ariance ratio } & $2 \cdot 509^{*}$ & 1.094 & $6.155 \dagger$ & 1.060 & & & & \\
\hline \multicolumn{2}{|l|}{$\begin{array}{l}\text {.F. } n_{1} \\
n_{2}\end{array}$} & $\begin{array}{r}6 \\
80\end{array}$ & $\begin{array}{r}5 \\
80\end{array}$ & $\begin{array}{r}5 \\
80\end{array}$ & $\begin{array}{r}80 \\
5\end{array}$ & & & & \\
\hline
\end{tabular}

The results of all the experiments to which the equation could be fitted, were those for which the inoculum was either crude sap or purified virus preparations at concentrations not exceeding $1 \mathrm{~g}$./1. The equation was fitted on the assumption 
that when the virus concentration was somewhere in the range $0.2-1.0 \mathrm{~g} . / \mathrm{l}$., all the accessible susceptible regions developed lesions. Now, according to the assumptions from which equation $(1 a)$ was derived, there is no gradation in susceptibility between susceptible regions, and only their number per half-leaf can vary. It follows that whenever a set of plants is inoculated with the same virus preparation, the maximum number of lesions should be obtained when the virus concentration reaches about $1 \mathrm{~g}$. $/ \mathrm{l}$. That this is not so, can be seen from the results of some of the experiments in which dilution-infection series were started from the virus concentration of $20 \mathrm{~g} . / 1$.

Table 7. Comparisons of experimental with computed values for Exp. no. 13

\begin{tabular}{|c|c|c|c|c|c|}
\hline \multirow{2}{*}{$\begin{array}{l}\text { TMV conc. } \\
\text { (g./l.) }\end{array}$} & \multirow{2}{*}{$Y=\phi \underset{\bar{z}}{(x) \rightarrow}$} & \multirow{2}{*}{$\begin{array}{c}I \\
(\mathbf{2}) \\
\boldsymbol{Z}\end{array}$} & \multirow{2}{*}{$\begin{array}{c}\text { II } \\
(\mathbf{4}) \\
\boldsymbol{Z}\end{array}$} & \multicolumn{2}{|c|}{ Parameters } \\
\hline & & & & $\mathbf{I}$ & II \\
\hline $20 \cdot 0$ & 2.3917 & $2 \cdot 3560$ & $2 \cdot 3628$ & $\xi=0.27$ & $a_{1}=\mathbf{3 3 5}$ \\
\hline $4 \cdot 0$ & $2 \cdot 3100$ & $2 \cdot 2780$ & $2 \cdot 3389$ & $\lambda=2 \cdot 16$ & $N_{1}=59$ \\
\hline 0.8 & $2 \cdot 1217$ & $2 \cdot 1774$ & $2 \cdot 1403$ & $N=329$ & $a_{2}=0.63$ \\
\hline $0 \cdot 16$ & 2.0275 & $2 \cdot 0443$ & 1.9648 & & $N_{2}=153$ \\
\hline 0.032 & 1.9092 & 1.9121 & 1.9065 & & \\
\hline 0.0064 & 1.7908 & 1.7580 & 1.8527 & & \\
\hline 0.00128 & 1.5742 & 1.6100 & 1.5936 & & \\
\hline 0.000256 & 1.4817 & 1.4741 & $1 \cdot 3696$ & & \\
\hline$W \rightarrow$ & & 5 & 4 & & \\
\hline$\frac{n}{W} \Sigma\left(\bar{z}_{i}-Z_{i}\right.$ & $i)^{2} \rightarrow$ & 0.0194 & 0.0682 & & \\
\hline Variance $r$ & atio & 1.474 & $2 \cdot 385$ & Neither ratio is & significant at \\
\hline $\begin{array}{r}\text { D.F. } n_{1} \\
n_{2}\end{array}$ & & $\begin{array}{r}80 \\
5\end{array}$ & $\begin{array}{r}4 \\
80\end{array}$ & $\boldsymbol{P}=\mathbf{5} \%$ & \\
\hline
\end{tabular}

Equation (2) could be fitted approximately to the results of all the experiments obtained in this work, so the set of assumptions from which it was derived may be true. Yet Lauffer \& Price (1945) definitely reject these assumptions and accept those leading to equation $(1 a)$. They base their conclusions on three lines of evidence. The first is that some of the published data on tobacco mosaic virus on $N$. glutinosa are sufficiently accurate to show that they fit equation ( $1 a$ ) better than equation (2). They demonstrate this point graphically (Fig. 6 in their paper) with the results of two experiments selected from the literature, which they state fit equation ( $1 a$ ) better than equation (2). The data did not fit equation (2) as well as they did equation (1 $a$ ) only because the values of the parameter $N$ in equation $(1 a)$ were adjusted to obtain a good fit to the data, and then the same values were used for the parameter $N$ in equation (2). The parameter $\lambda$ was taken as 0.5. A much better fit is obtained with different values for $N$ and $\lambda$.

The second line of evidence given by Lauffer \& Price is that 'the dilution curves of all viruses (of animals and plants) tend to have the same slope', notably the slope of the curve of equation $(1 a)$. The slope of the curve of equation $(1 a)$ is characteristic at each point corresponding to any particular 
value of $a x$, whereas the slope of equation (2) is not characteristic at any particular point unless there is a reason for fixing the parameter $\lambda$ at some particular value for all viruses, and there does not seem to be any such reason. However, it was because the slope of the line of experimental points could not be made to coincide with the curve of equation ( $1 a$ ) that equation ( $1 a$ ) could not be fitted to the data of many experiments with tobacco mosaic virus, whereas the curve of equation (2) could be fitted to the data of different experiments by substituting for $\lambda$ different values considerably removed from 0.5 , at which the slope of the curve most closely approaches that of the curve of equation $(1 a)$.

The third line of evidence used by Lauffer \& Price comes from the study of local infections caused by mixed inocula. When a mixture of the related strains of tobacco mosaic and aucuba mosaic viruses is inoculated to $N$. langsdorffi, on which both form necrotic local lesions, some lesions appear to contain both strains, and some only one or the other. If the assumptions from which equation $(1 a)$ is derived are true, and if each virus strain can multiply freely in the presence of the other, the proportion of mixed infections can be predicted. If the two strains are mixed in equivalent proportions, i.e. if each strain, when present alone, forms approximately the same number of lesions, and if the parameters $N$ and $a$ in equation ( $1 a$ ) are adjusted so that the equation fits the results of dilution-infection series with the mixed inoculum, the proportion of lesions containing both strains obtained with a total virus concentration $x$ will be expected to be given by the value of the infinite series

$$
\rho=\frac{e^{-a x}}{1-e^{-a x}}\left\{\left[1-\frac{1}{2}\right] \frac{a^{2} x^{2}}{2 !}+\left[1-\left(\frac{1}{2}\right)^{2}\right] \frac{a^{3} x^{3}}{3 !}+\left[1-\left(\frac{1}{2}\right)^{3}\right] \frac{a^{4} x^{4}}{4 !}+\ldots\right\} .
$$

The experimental results obtained by Lauffer \& Price differ widely from computed expectations, so that it seems strange that they should have considered them as evidence in favour of the assumptions on which the expectations are based. The computation is based on the assumption that each virus strain can multiply freely in the presence of the other, whereas there is much evidence to the contrary. If one virus strain is established in a host plant, it prevents the subsequent development of another related strain, and when two related strains are inoculated simultaneously, one can interfere with the formation of local lesions by the other (Sadasivan, 1940). Thus the presence of only one virus strain in a local lesion may be a result of suppression of development of the other strain. If so, the results obtained by Lauffer \& Price cannot be considered as evidence for or against either of the two hypotheses under consideration.

The conclusion arrived at in this study, that the set of assumptions from which equation $(1 a)$ was derived must be false, does not exclude the possibility that the one virus particle in a susceptible region might produce a lesion. If equation $(1 a)$ is modified by changing the original assumptions or by introducing some auxiliary assumptions, it may then be fitted satisfactorily to the experimental results. One such modification, based on the assumption that virus particles are aggregated and dissociate on dilution, was introduced by 
Bald $(1987 \mathrm{c})$. However, Bald's interpretation of the fact that the modified equation ( $1 a$ ) could be fitted satisfactorily to the experimental results, is that it 'is no proof that the kind of aggregation postulated in the derivation of the (modified) equation actually exists, and the constant $K$ (present in the modified equation) must be considered as a measure of distortion of the dilution series rather than a measure of aggregation, unless an independent proof is obtained that the virus particles do aggregate in this manner'. Particles of tobacco mosaic virus can occur in linear aggregates, and the extent of such aggregation varies from one preparation to another depending mainly on the treatments to which the preparation has been subjected (Bawden \& Pirie, 1945; Crook \& Sheffield, 1946). There is no evidence that these aggregates dissociate on dilution, and no reason to assume that they would do so to the extent that would have to be assumed to make equation $(1 a)$ fit the experimental data. To fit equation ( $1 a$ ) to the results of Exp. no. 18, for example, it would have to be assumed that for the range of concentrations between 2.0 and $0.002 \%$, one virus aggregate is broken on average into 2.6 every time the solution was diluted 1:5. Also, as Fig. 5A shows, to fit equation $(1 a)$ to the results of different experiments, it is necessary to assume that the disaggregation caused by diluting the same virus preparation differs at different times.

The original assumptions from which equation $(1 a)$ was derived can be modified in other ways to fit the experimental results. Any modification that would make the parameter $a$ vary suitably from one susceptible region to another, would serve this purpose. For example, it was originally assumed that the volume of inoculum that comes into contact with any susceptible region, is approximately constant, but if it is not, the value $a$ will also vary. Alternatively, it can be assumed that there are $u$ kinds of virus particles differing from one another in such a way that a particle of one kind can effectively infect any of $u$ kinds of susceptible regions, a particle of the second kind can infect any of $(u-1)$ kinds of regions, a particle of the third kind any of $(u-2)$ kinds of regions, and so on. Any of these assumptions would modify equation ( $1 a$ ) into the form

$$
Y=\sum_{i-1}^{u} N_{i}\left(1-e^{-a_{i} x}\right)
$$

In fact, if only two different values for $N$ and $a$ are postulated, so that equation ( $1 a$ ) will be modified into equation (4), it could be fitted satisfactorily to the results of all the experiments obtained in this work by suitably adjusting the values of the four parameters $N_{1}, a_{1}, N_{2}$ and $a_{2}$. This was shown statistically for Exp. 13. The fact that adding various auxiliary assumptions to the hypothesis, that infection can be caused by single virus particles, can make it compatible with experimental results, proves nothing, but it does make it possible that the hypothesis may be true.

Equation (2) could be approximately fitted to the results of all the experiments obtained in this work. Thus the hypothesis of variation in susceptibility of susceptible regions, with logarithms of minimal effective concentrations normally distributed, may be true. The fact that no auxiliary assumptions are needed may be considered as an argument in favour of the hypothesis. Any 
conclusive evidence is to be expected from some new experimental approach to the problem rather than from any mathematical treatment of the information at present available.

In conclusion it should be emphasized that there is no reason to suppose that all infective agents referred to as 'viruses' would display the same basic mechanism of infecting their hosts, as it is often assumed in discussions on the subject.

\section{REFERENCES}

BALD, J. G. (1937 a). The use of numbers of lesions for comparing the concentrations of plant virus suspensions. I. Dilution experiments with purified suspensions. Ann. appl. Biol. 24, 33.

BaID, J. G. (1937b). The use of numbers of lesions for comparing the concentrations of plant virus suspensions. II. Distortion of the dilution series. Ann. appl. Biol. 24, 56.

BALD, J. G. (1937c). The use of numbers of lesions for comparing the concentrations of plant virus suspensions. IV. Modification of the simple dilution equation. Austr. J. exp. Biol. med. Sci. 15, 211.

Bawden, F. C. \& Pirie, N. W. (1945). The isolation and properties of tobacco mosaic virus in different states of aggregation. Brit. J. exp. Path. 26, 294.

Burss, C. I. (1935). The calculation of the dosage-mortality curve. Ann. appl. Biol. 22, 134 .

Bryan, W. R. \& Brard, J. W. (1940). Host influence on the characterization of response to the papilloma protein and to vaccinia virus. J. infect. Dis. 67, 5.

Crook, E. M. \& Sherfield, F. M. L. (1946). State of aggregation of tobacco mosaic virus. Brit. J. exp. Path. 27, 328.

Finney, D. J. (1947). Probit Analysis. Cambridge University Press.

GADDUM, J. H. (1933). Methods of biological assay depending on a quantal response. Spec. Rep. Ser. Med. Res. Coun. no. 183. London: H.M. Stationery Office.

Haldane, J. B. S. (1939). Sampling errors in the determination of virus density by the dilution method. J. Hyg., Camb., 39, 289.

Kr.eCZkowski, A. (1949). The transformation of local lesion counts for statistical analysis. Ann. appl. Biol. 36, 139.

Laufrer, M. A. \& Price, W. C. (1945). Infection by viruses. Arch. Biochem. 8, 449.

Parker, R. F. (1988). Statistical studies of the nature of the infectious unit of vaccinia virus. J. exp. Med. 67, 725 .

Sadasivan, T. S. (1940). A quantitative study of the interaction of viruses in plants. Ann. appl. Biol. 27, 359.

Youden, W. J., Beale, H. P. \& Guthrie, J. D. (1935). Relation of virus concentration to the numbers of lesions produced. Contr. Boyce Thompson Inst. 7, 37. 